\title{
Trajectory planning for a planar macro-micro manipulator of a laser-cutting machine
}

\author{
Emre Uzunoglu, Mehmet Ismet Can Dede and Gökhan Kiper \\ Department of Mechanical Engineering, Izmir Institute of Technology, Izmir, Turkey
}

\begin{abstract}
Purpose - In the industry, there is always a demand to shorten the task completion durations to maximize the efficiency of the operation. This work focuses on making use of a special type of kinematic redundancy, macro-micro manipulation, to minimize the task completion duration. The purpose of this paper is to develop the most convenient trajectory planner to be integrated with industrial computerized numerical control (CNC) systems to resolve kinematic redundancy for task duration minimization.

Design/methodology/approach - A special type of kinematic redundancy is devised by using two kinematically different mechanisms that have different advantages, which are named as macro and micro mechanisms. In this case, the control design including the trajectory planning should be devised taking into account the distinct advantages of both mechanisms. A new trajectory planning algorithm is designed and used for the constructed planar laser-cutting machine, and some benchmark pieces are cut.

Findings - Offline method has practical limitations for employment in a real case scenario such as assuming infinite jerk limits for each axis motion. This limitation was removed by using an online trajectory generation technique. Experimental test results indicate that the online trajectory planning technique developed for the macro-micro mechanism to shorten the task duration was successful.

Practical implications - Although the new trajectory planning algorithm is implemented for a laser-cutting machine, it can also be used for other manufacturing systems that require higher acceleration and accuracy levels than the conventional machines. The new algorithm is compatible with the commercially available CNC systems.

Originality/value - In this work, a new approach to reducing the task duration for planar machining operations was introduced by making use of macro-micro manipulation concept. The core novelty of the work is devising trajectory planning algorithms to get the most efficiency in terms of acceleration limits from a macro-micro manipulation while making these algorithms deployable to most of the CNC systems.
\end{abstract}

Keywords Macro-micro manipulator, Redundancy resolution, Redundant manipulator, Trajectory planning

Paper type Research paper

\section{Introduction}

Automation in the manufacturing industry has proved to provide precise and faster manufacturing results in years. Introduction of new technologies in tool design pushed the limits of manufacturing systems further. Among these tools, fibre laser technology found application in planar and three-dimensional (3D) cutting and welding operations. As it can deploy relatively large amount of focused energy, fibre laser technology has been selected for high precision and time-efficient manufacturing systems (Hügel, 2000).

Although fibre laser cutting is one of the fastest technologies for especially planar cutting operation of sheet metals and plastics, there is still a demand from the manufacturing industry to decrease the manufacturing task completion durations. At the current state of fibre laser-cutting operation, cutting speed is mostly determined by the laser power source, material type and the width of the material (Steen and Mazumder, 2010). Therefore, to

The current issue and full text archive of this journal is available on Emerald Insight at: www.emeraldinsight.com/0143-991X.htm

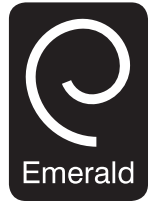

Industrial Robot: An International Journal

43/5 (2016) 513-523

C Emerald Group Publishing Limited [ISSN 0143-991X]

[DOI 10.1108/IR-02-2016-0057] exceed the conventional laser cutting machine's task completion duration, the only option without changing the composition of the tool is to reach the maximum cutting speed as fast as possible. This strategy can gain much time especially for the cases in which there are relatively large number of small contours and sharp edges defined for the cutting path of the tool.

Usually, conventional planar laser-cutting machines are built to cover the whole sheet metal area, and therefore, their workspace is relatively larger, which is usually in the range of 1-2 $\mathrm{m}$ by 2-3 $\mathrm{m}$. During the cutting process of workpiece with many small contours, mostly the maximum allowable tool speed is never reached by the conventional machines, as it has limited acceleration due to large inertial properties of the construction that is moving the tool in $x$ - and $y$-axes. In the case of using larger actuators with greater torque capabilities, it can be foreseen that the system will lose precision while increasing the acceleration capability. As a consequence of these facts, generally,

This study is granted by Republic of Turkey Ministry of Science, Industry and Technology and Coşkunöz Metal Form Inc. (Project code: 01,668.STZ.2012-2). The authors thank to Coşkunöz who provided the test equipment and Mr Ercan Mastar from Coşkunöz for his collaboration and valuable support.

Received 1 February 2016

Revised 20 March 2016

Accepted 19 April 2016 
acceleration limits of the conventional planar laser-cutting machines are set at 1-1.5 g.

One solution to go beyond the acceleration limits set by the conventional machines is by introducing kinematic redundancy to the conventional machine to take advantage of optimization of redundant manipulation. Although there have been numerous studies for redundancy concept in robot manipulators, in many of them, kinematic redundancy is realized by the addition of an extra link of the same kind to a serial kinematic chain (Nakamura, 1991). A unique set of kinematic redundancy can be formed by the use of different mechanisms that inherently have distinct capabilities. Macro-micro manipulation is a member of this unique set by having a macro mechanism with relatively larger workspace and a micro mechanism with relatively more dynamic behaviour. To the best knowledge of the authors, the macro-micro manipulation idea was explicitly used for the first time in the work of Marzwell et al. (1994). In their work, a surgery robot with macro-micro manipulation was studied. Micro mechanism of this surgery robot was used for force control at higher bandwidth, and course positioning was done by the macro mechanism. In terms of laser-cutting operation, while the macro mechanism can be used to provide the coverage of the large workspace in planar laser-cutting applications, the high acceleration limits of the micro mechanism can be used for increasing the tool's acceleration; hence, the workspace is not shrunk, and the task completion duration can be reduced. A general representation of a planar macro-micro manipulator is presented in Figure 1. Macro-micro manipulator systems for planar laser-cutting applications were patented by Sartorio (2004), Leibinger et al. (2004), Cardinale et al. (2007) and Battheu (2011).

Obviously, an intelligent trajectory planning algorithm should be devised to take advantage of the macro-micro manipulation. In this work, our aim is to present a method to distribute a planar motion path, which is described for laser cutting, to the trajectories of the macro and micro mechanisms to complete the task in a minimum duration. The main limitation of this work is that the developed algorithm should be applicable to industrial computerized numerical control (CNC) systems. Therefore, in the streamline of a computer integrated manufacturing system, this algorithm is placed as the post-processor after the numerical control programming language codes (G-codes) are generated by use of a computer-aided-manufacturing program.
In the next section, previous work on trajectory planning of macro-micro manipulators is presented along with our previous work on this specific manufacturing system. Our new trajectory planner is explained in detail in the following section which is supported by verification of the new algorithm by simulation and experimental results. The paper is finalized with discussions and conclusions.

\section{Previous work on macro-micro mechanism trajectory planning}

Much research has been focused on optimizing the trajectory of kinematically redundant manipulators by taking advantage of infinite number of solutions while achieving the same primary task. Among these, self-motion of the redundant manipulator, as termed by Nakamura (1991), is used to achieve subtasks. Redundancy resolution to distribute the total motion to redundant axes in velocity level by making use of pseudo-inverse approaches has been widely used in regulating self-motion for singularity avoidance by Yoshikawa (1984), joint velocity minimization by Seraji (1991), obstacle avoidance by Chen et al. (2002), mechanical joint limit avoidance by Tatlicioglu et al. (2009) and manipulability by Maaroof et al. (2012). The commonality of these approaches is that the extra links that make the manipulator redundant are similar in term of kinematic and dynamic capabilities of the previous links. However, macromicro manipulators, which are a subset of kinematically redundant manipulators, are a combination of two mechanisms with distinct kinematic and dynamic properties. Therefore, the redundancy resolution algorithm should be formed with respect to this fact to take the most advantage of the distinct behaviour of the two mechanisms.

A dedicated redundancy resolution algorithm can be formulated for macro-micro manipulators depending on the task and the composition of the mechanisms. Several control approaches have been proposed in literature that benefits the macro-micro manipulation for a variety of tasks, i.e. for increasing accuracy, reducing inertia effects or manipulability. To reduce the effect of inertia, a dextrous dynamic control of macro-micro manipulator is proposed by Khatib (1991), where the manipulator redundancy is used for decreasing the deviations of micro-mechanism's joints. Macro-micro manipulation with a robust controller based on physical equivalence and impedance matching was proposed by Sharon et al. (1993). In another study by Nagai and

Figure 1 Planar macro-micro mechanism concept

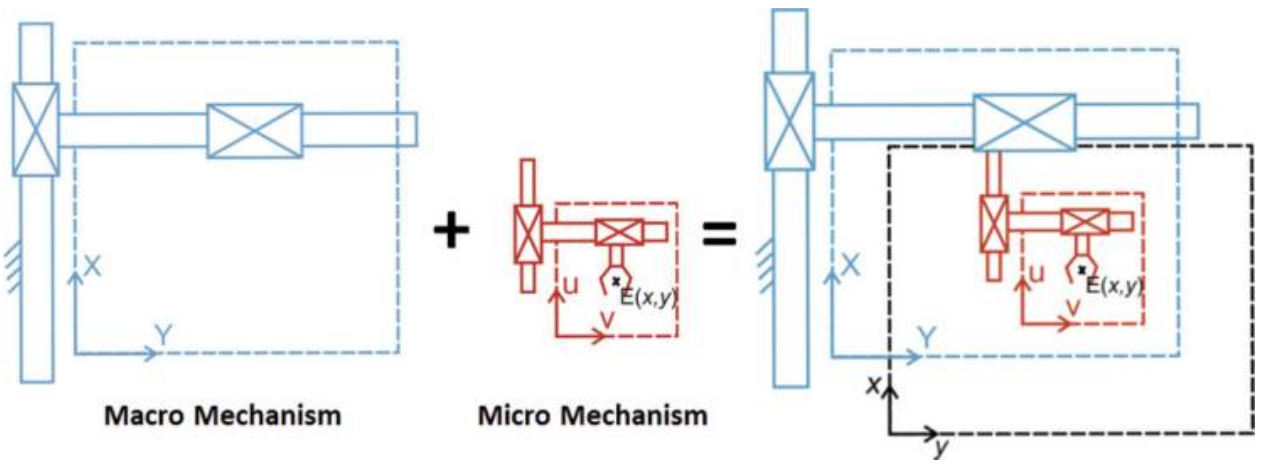


Yoshikowa (1994), an impedance controller is adapted for redundant macro-micro manipulator in which not only the desired mechanical impedance is controlled but also the internal force applied at the tip of the macro manipulator is considered. A method is developed by Sadigh and Salehi (2004) that have an outer loop control, based on artificial constrained motions, to eliminate deviations. In the study of Quan et al. (2006), motions are generated with the relation between kinetic energy and manipulability of macro-micro mechanism, which is developed for imitating the hand and arm movement of a human.

In the scope of this work, the major concern is to increase the acceleration limits for the macro-micro manipulator's trajectory to be developed for the tool path while keeping the acceleration of the macro mechanisms limited at $1 \mathrm{~g}$. The limitation for the macro mechanism is set to avoid high amplitude vibrational behaviour due to large inertia of the macro mechanism, which otherwise would have resulted in imprecise motion. The total acceleration limit aimed for the tool trajectory is set at $6 \mathrm{~g}$, while the workspaces of the macro and micro mechanisms are limited as $3 \mathrm{~m} \times 1.5 \mathrm{~m}$ and $0.1 \mathrm{~m} \times 0.15 \mathrm{~m}$, respectively. The details on our macro-micro mechanism design is shown in Figure 2, where macro and micro mechanism's axes are denoted, respectively, by $\mathrm{X}-\mathrm{Y}$ and $\mathrm{u}-\mathrm{v}$. In our previous works that appeared in (Uzunoğlu et al., 2014) and (Uzunoğlu et al., 2015), we formulated two different trajectory planning solutions for this macro-micro mechanism.

Main difference of our previously developed algorithms is that one of them works as an offline post-processor (Uzunoğlu et al., 2014) while in the other one, the trajectory for the micro mechanism is generated online (Uzunoğlu et al., 2015). However, in both methods, generated G-codes for the tool path behaviour are used initially. The offline method is based on the patent of Sartorio (2004) and explained in detail in (Uzunoğlu et al., 2014). Once the trajectory segments are distinguished as travelling and machining segments, the velocity limit is determined for each segment. In travelling segments, the tool is moved to the next machining segment with the velocity limit of the macro mechanism set as the velocity limit of the tool. In machining segments, tool is operated for machining and therefore, the velocity limit is determined with respect to the maximum machining limit with respect to the laser power, workpiece material and thickness. In developing the trajectories of both macro and micro mechanisms to reach these velocities in both segments, maximum combined acceleration and deceleration is set as $6 \mathrm{~g}$. In this way, the trajectories for both mechanisms are

Figure 2 Macro-micro laser-cutting machine design denoting each mechanism's axes

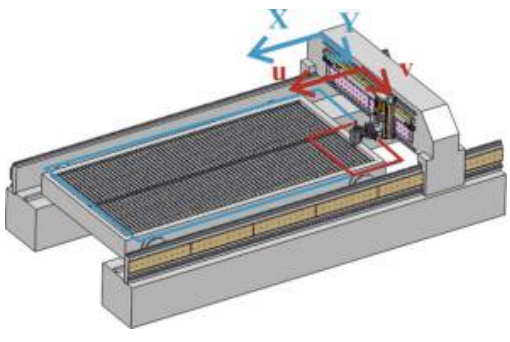

determined offline and then it can be operated by the CNC system. The practical problem with this approach is that at certain times the jerk of each mechanism is set as infinite, which cannot be satisfied by the actual system, and thus, it would result in unprecise machining.

The trajectory planning algorithm which includes online trajectory generation for the micro mechanism was initially developed by increasing the acceleration limits for the tool trajectory by offline planning. Then, by using corner reduction and smoothing algorithms, the trajectory of the macro mechanism is developed offline. Finally, the difference between the tool trajectory and the macro mechanism motion is calculated online and provided as an online trajectory for the micro mechanism. This algorithm is explained in detail in Uzunoğlu et al. (2015) and based on this, our new algorithm is explained in the next section.

\section{New trajectory planning algorithm to be deployed on CNC}

The new trajectory generation algorithm is developed to expose the capabilities of both macro mechanism or otherwise called as primary mechanism (PM), which operates in $x-y$-axes and micro mechanism or otherwise called as secondary mechanism (SM), which operates in $u$-v-axes. In this new algorithm, the continuous and smoothened motion (motion never coming to a full stop) is derived for macro mechanism's trajectory in global space while high acceleration motions are reserved for the micro mechanism. This is the offline part of trajectory planning algorithm in which the output is the jerk-bounded trajectory of the macro mechanism. Macro mechanism's trajectory is now ready to be used in the CNC machine controller in terms of time series data with a sampling rate of $0.5 \mathrm{~ms}$. This sampling rate is determined with respect to the capabilities of the CNC used in this work. Flowline of trajectory information is given in Figure 3. Each step of the trajectory generation algorithm is explained in the following subsections.

\subsection{Offline trajectory generation algorithm}

Offline trajectory generation flow starts with registering function inputs. The necessary inputs consist of segment data extracted from G-codes and manually entered inputs, which are the motion limitations. As the motions are generated in jerkbounded S-shaped profiles, both acceleration and jerk limits should be specified within motion limitations. Moreover, the function enables inputting different limitations for travelling, cutting and leading/piercing processes. After referencing the workpiece inside the workspace of the machine, initial coordinates should also be entered as an input with respect to the global workspace. In this study, no leading or piercing process is used, but the developed algorithm allows the user to enter piercing time or leading if required.

The segment data are obtained from G-codes for trajectory generation and converted to sequential format in the "G-code to segmentation interpreter" function block. This sequential format is later used as input in "Filtering Function" function block. The segment data received from the G-code in the array 
Figure 3 Flowline of the new trajectory generation algorithm



format are in the form $[N G M X Y Z I F]$ in which the letters in the array denote:

$N$ : \# of line.

$G$ : Contour description (0: Travelling, 1: Line cutting, 2: CW cutting, 3: CCW cutting).

$M$ : Function (3: Laser on, 5: Laser off, 30: Program done, 34: Lead in, 35: Lead out, 4: Piercing).

$X, Y, Z: \mathrm{x}, \mathrm{y}$ and $\mathrm{z}$ motion coordinates.

$I, \mathcal{F}$ : Circular motion centre coordinates.

Filtering function is the main novelty of the work presented in this paper which has a vital role in trajectory generation process that generates motions in time series to be transferred to the actuators. The first step of this process is generating tool trajectory and deriving the macro mechanism's motion using filtering function to the time series data.

Trajectory of the tool is planned in S-shaped segments with a time step of $0.5 \mathrm{~ms}$. Travelling process is divided into seven phases with respect to distance $S(t)$, velocity $V(t)$, acceleration $A(t)$ and jerk $\mathcal{f}(t)$. In Figure $4, T_{i}$ is the total time of each phase, $S$ is the total distance travelled, $V$ is the velocity, $A$ is the acceleration and $\mathcal{F}$ is the jerk. The phases are the increasing acceleration phase $\left(T_{1}\right)$, the constant acceleration phase $\left(T_{2}\right)$, the decreasing acceleration phase $\left(T_{3}\right)$, the constant velocity phase $\left(T_{4}\right)$, the decreasing deceleration phase $\left(T_{5}\right)$, the constant deceleration phase $\left(T_{6}\right)$ and the increasing deceleration phase $\left(T_{7}\right)$.

The expression for calculating the motions in acceleration, velocity and displacement levels are presented equations (1), (2), and (3). $t_{i}-$ the terminating duration of stage $T_{i}$, where $i=0,1[\ldots], 7$. $\tau_{i}-$ the relative time within the phase, where $i=0,1[\ldots], 7$.

$$
\begin{aligned}
& A(t)=\left\{\begin{array}{cl}
\mathfrak{F} \tau_{1} & 0 \leq t<t_{1} \\
\mathfrak{F} T_{1} & t_{1} \leq t<t_{2} \\
\mathfrak{F} T_{1}-\mathfrak{F} \tau_{3} & t_{2} \leq t<t_{3} \\
0 & t_{3} \leq t<t_{4} \\
-\mathfrak{F} \tau_{5} & t_{4} \leq t<t_{5} \\
-\mathfrak{F} T_{5} & t_{5} \leq t<t_{6} \\
-\mathfrak{F} T_{5}+\mathfrak{F} \tau_{7} & t_{6} \leq t<t_{7}
\end{array}\right. \\
& V(t)=\left\{\begin{array}{cc}
V_{i}+\mathfrak{F} \tau_{1}^{2} / 2 & 0 \leq t<t_{1} \\
V_{1}+\mathcal{F} T_{1} \tau_{2} & t_{1} \leq t<t_{2} \\
V_{2}+\mathfrak{F} T_{1} \tau_{3}-\mathfrak{F} \tau_{3}^{3} / 2 & t_{2} \leq t<t_{3} \\
V_{3} & t_{3} \leq t<t_{4} \\
V_{4}-\mathfrak{F} \tau_{5}^{2} / 2 & t_{4} \leq t<t_{5} \\
V_{5}-\mathcal{F} T_{5} \tau_{6} & t_{5} \leq t<t_{6} \\
V_{6}-\mathfrak{F} T_{5} \tau_{7}+\mathfrak{f} \tau_{7}^{2} / 2 & t_{6} \leq t<t_{7}
\end{array}\right.
\end{aligned}
$$

where:

$$
\begin{aligned}
& V_{1}=V_{i}+\mathfrak{F} T_{1}^{2} / 2, \\
& V_{2}=V_{1}+\mathcal{F} T_{1} T_{2}, \\
& V_{3}=V_{2}+\mathfrak{F} T_{1} T_{3}-\mathcal{F} T_{3}^{2} / 2, \\
& V_{4}=V_{\max }, \\
& V_{5}=V_{4}-\mathfrak{F} T_{5}^{2} / 2, \\
& V_{6}=V_{5}-\mathfrak{F} T_{5} T_{6} .
\end{aligned}
$$

$$
S(t)=\left\{\begin{array}{cc}
V_{i} \tau_{1}+\mathfrak{F} \tau_{1}^{3} / 6 & 0 \leq t<t_{1} \\
S_{1}+V_{1} \tau_{2}+\mathfrak{F} T_{1} \tau_{2}^{2} / 2 & t_{1} \leq t<t_{2} \\
S_{2}+V_{2} \tau_{3}+\mathfrak{F} T_{2} \tau_{3}^{2} / 2-\mathfrak{F} \tau_{3}^{3} / 6 & t_{2} \leq t<t_{3} \\
S_{3}+V_{3} \tau_{4} & t_{3} \leq t<t_{4} \\
S_{4}+V_{4} \tau_{5}-\mathfrak{F} \tau_{5}^{3} / 6 & t_{4} \leq t<t_{5} \\
S_{5}+V_{5} \tau_{6}+\mathfrak{F} T_{5} \tau_{6}^{2} / 2 & t_{5} \leq t<t_{6} \\
S_{6}+V_{6} \tau_{7}-\mathfrak{F} T_{5} \tau_{7}^{2} / 2-\mathfrak{F} \tau_{7}^{3} / 6 & t_{6} \leq t<t_{7}
\end{array}\right.
$$

where:

$$
\begin{aligned}
& S_{1}=V_{i} T_{1}+\mathfrak{F} T_{1}^{3} / 6, \\
& S_{2}=S_{1}+V_{1} T_{2}+\mathfrak{F} T_{1} T_{2}^{2} / 2, \\
& S_{3}=S_{2}+V_{2} T_{3}+\mathfrak{F} T_{2} T_{3}^{2} / 2-\mathfrak{F} T_{3}^{3} / 6, \\
& S_{4}=S_{3}+V_{3} T_{4}, \\
& V_{5}=S_{4}+V_{4} T_{5}-\mathfrak{F} T_{5}^{3} / 6, \\
& S_{6}=S_{5}+V_{5} T_{6}+\mathfrak{F} T_{5} T_{6}^{2} / 2 .
\end{aligned}
$$

The motion profile for each travelling distance is calculated and time series data are generated within the combined macro-micro mechanism's limits. Motion profiles may not have constant velocity or constant acceleration phases in short travelling ranges which is accounted for in the algorithm.

The aim of the filtering function is to derive the trajectory of the macro mechanism's axes which should have slowly varying velocity profile. As a result of jerk-bounded motion planning for the input in the form of a segment data, a time series of data $S(t), t=0$ [. .], $n$, is generated for the trajectory of the tool path. The tool trajectory, $S(t)$, is formulated to have both motions with slowly varying velocity for macro mechanism, $X(t)$, and faster varying velocity with relatively small displacements for micro mechanism, $U(t)$. When $X(t)$ is 
Figure 4 Distance, velocity, acceleration and jerk profiles of S-shaped motion planning
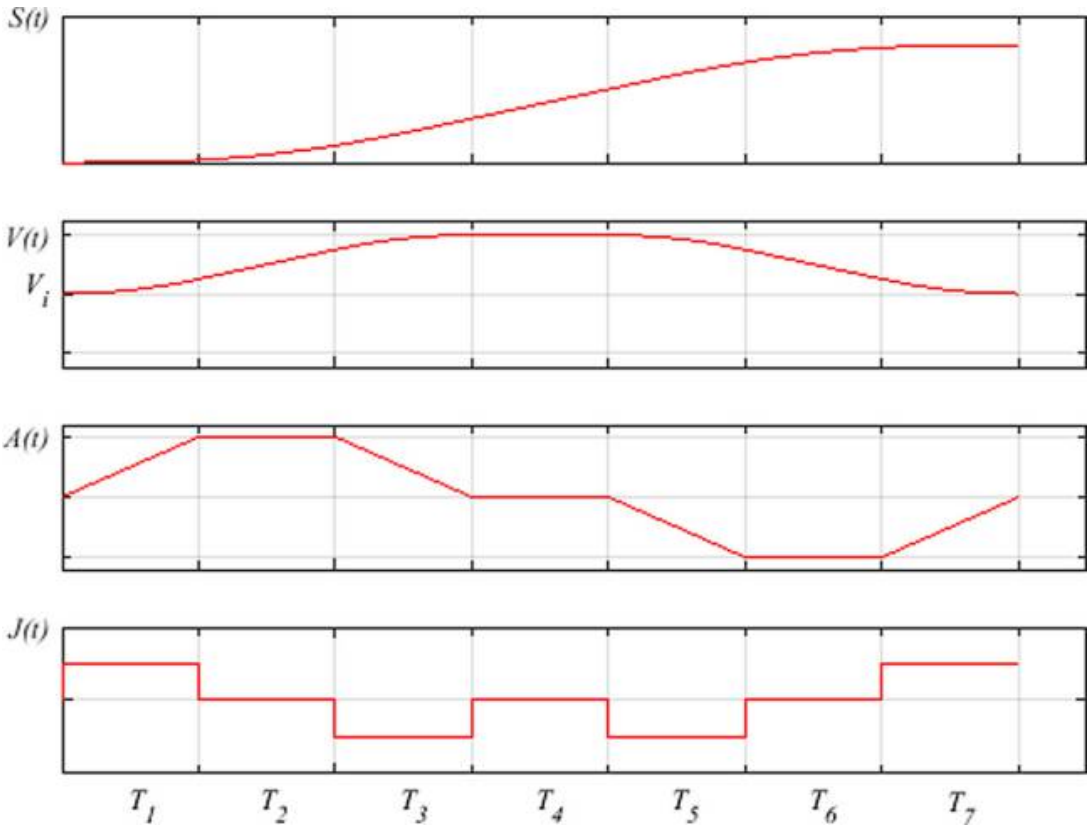

extracted from $S(t)$, then the macro mechanism will have a smooth and continuous motion.

To extract the smoothened component of the tool trajectory, $X(t)=S(t)-U(t)$, Hodrick-Prescott filtering is adapted (Kim et al., 2009). This filtering technique has been used mainly in macro scale time series analysis. In this technique, $X(t)$ is derived by minimizing the weighted sum objective function represented in equation (4).

$$
\frac{1}{2} \sum_{t=0}^{n}(S(t)-X(t))^{2}+\lambda \sum_{t=1}^{n-1}(X(t-1)-2 X(t)+X(t+1))^{2}
$$

In equation (4), $\lambda>0$ is the weighing parameter used to regulate the trade-off between smoothness of $X(t)$ and the size of the residual $U(t)$. The size of the residual should be kept inside the workspace limitation of the micro mechanism. The trend value, $\lambda$, is selected after three iterations for every time the filtering function runs, in which the workspace limitation of the micro mechanism and acceleration limits of both mechanisms are checked whether they are within the range of the related physical capabilities of both mechanisms. The change in the workspace of the micro mechanism also changes the maximum acceleration expectations from the micro mechanism during the operation. The superiority of the proposed method with respect to the previously developed methods is that this tuning capability brings much flexibility in distributing the accelerations between the macro and micro mechanism. As the acceleration limits of the macro mechanism is decreased by this tuning capability, the precision (accuracy) of the total system can be improved.

As a result of the offline trajectory generation (pre-processing) part of the algorithm, two-time series of data are generated, one for the macro mechanism ( $X-Y$ axes) and one for the tool. The sampling rate of trajectories is chosen as $2 \mathrm{kHz}$, which corresponds to the maximum frequency that $\mathrm{CNC}$ controller can feed the actuator commands to the drivers of the servo motors.

\subsection{Online trajectory tracking algorithm}

After the offline trajectory generation phase, the generated data are fed to the online process which directs the motion commands to the CNC controller that includes the drivers for the servomotors actuating all the axes. The smoothened macro mechanism's motion commands are directly used as inputs to the drivers that actuate the macro mechanism's servomotors. On the other hand, the micro mechanism's motion commands are to be calculated in a higher level control loop before delivering these commands to the drivers of the micro mechanism's servomotors.

This semi-online method is devised to compensate for the offsets in tool trajectory that may occur in between the macro mechanism's motion and tool trajectory. Motion demands for the micro mechanism in $u$-v-axes are generated online by calculating offset, $U(t)=S(t)-X(t)$, in real time between the tool trajectory and the macro mechanism's measured position via absolute position encoders. This offset forms as a result of exclusion of the sharp edges in the smoothened motion trajectory of the macro mechanism and possible control errors of the macro mechanism axes. The motion demands calculated for $u$-v-axes is later converted to the joint motion commands using the inverse kinematics function developed for the micro mechanism's parallel robot structure. Inverse kinematics calculations and calibration process of the micro mechanism is explained in Uzunoğlu et al. (2015) and Kiper et al. (2015).

The trajectory generation algorithm was designed and tested first in simulations using Matlab and Simulink software packages. To use the algorithms in the CNC, functions of the 
trajectory planning algorithm are compiled and embedded in a UNIX-based real time operating system (RTLinux). The test results with the constructed system working with the CNC system are presented in the experimental results section.

\section{Experimental results}

As an implementation and verification, the proposed algorithm is used in a macro-micro mechanism planar laser-cutting machine to cut a benchmark piece that has a total of 100 consecutive holes with a $3 \mathrm{~mm}$ diameter, which are placed with $4 \mathrm{~mm}$ offset from each other. The experimentation is conducted on a galvanized steel sheet with $3 \mathrm{~mm}$ thickness as the work piece. To compare the developed method, same benchmark is cut with the conventional cutting procedure by using only the macro mechanism's axes. In this scenario, the micro mechanism's axes are locked at their homing position.

In the experiments, the planar macro-micro mechanism laser-cutting machine that was constructed within the scope of the same project is used. The details of the design of this machine are provided in Dede et al. (2014). Complying with the limitations of the experimental system, the acceleration of the PM's axes are limited with $9.81 \mathrm{~m} / \mathrm{s}^{2}$, the maximum acceleration of the secondary mechanism is set to $49.05 \mathrm{~m} / \mathrm{s}^{2}$, and the tool velocity is limited with $40 \mathrm{~m} / \mathrm{min}$, which is the maximum cutting speed due to the laser power and material specifications.

As proposed in the algorithm, the tool trajectory generated for benchmark cut is illustrated in Figure 5 with blue lines. This trajectory is generated from the tool path developed from G-codes complying with the motion limitations explained above. After the tool trajectory is generated, it is processed with the filtering function that is described in the previous section. The macro mechanism's trajectory is indicated in red colour in Figure 5. It can be clearly observed that the red line has no sharp edges when the tool is cutting out the square outer boundary. Also, the macro mechanism flows on top of the circular cuts without following the circle trajectories, which means that the circular trajectories are filtered out to be executed by the micro mechanism.

In Figure 6, motion demands for benchmark piece along two planar axes are shown. The motion demand for the macro mechanism's $x$ - and $y$-axes are indicated with red colour and the tool trajectories $(x-u)$ and $(y-v)$ are drawn in blue colour. A focused view is provided in Figure 6(b) to clearly show the smoothened trajectory of the macro mechanism. Relatively smaller displacements that can be observed in Figure 6(b) in blue colour are reserved for micro mechanism. The total task completion time is calculated as $26.04 \mathrm{~s}$ for 100 consecutive holes and their square frame contour.

After the pre-processing of G-codes and extracting the necessary motion demands both for the macro mechanism and the tool, these demands are exported to the online part of the algorithm. Micro mechanism's motion demands are calculated online by subtracting the measured macro mechanism's motion from previously generated time series data of the tool trajectory. In Figure 7, motion demands that are calculated online for the micro mechanism along $u$ - and $v$ - axes are given. These demands are processed online with the inverse kinematics formulation to calculate the motion demand for each actuated axis of the micro mechanism's parallel structure. After the benchmark piece is cut out, the measured trajectory of the tool is compared with the desired trajectory to calculate the error in trajectory following. These errors along each axis are given in Figure 8. It is observed that the errors are bounded within $\pm 0.5 \mathrm{~mm}$ range in both axes.

To compare the results in terms of improvement in task completion duration, same benchmark piece is cut by only using macro mechanism's axes which have acceleration limits at

Figure 5 Motion trajectories generated for benchmark piece (100 circle cut)

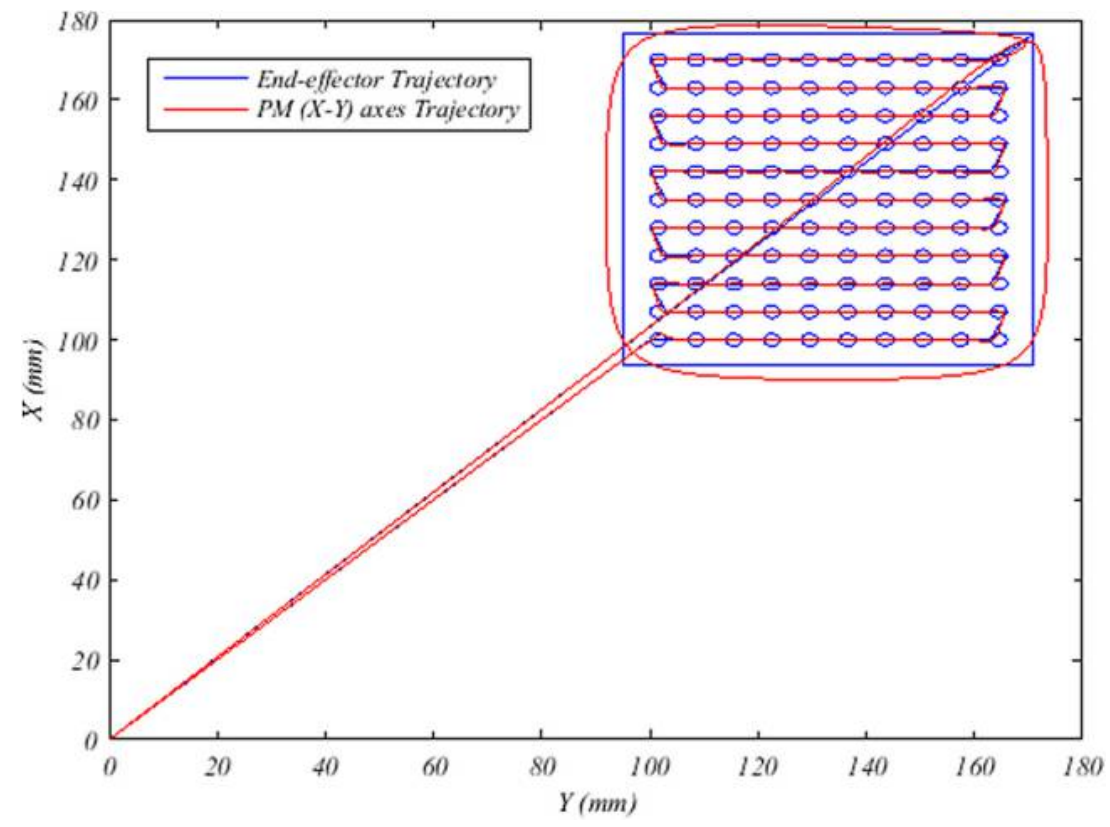


Figure 6 (a) Motion demands generated using proposed algorithm (b) Zoomed in version of the motion demands
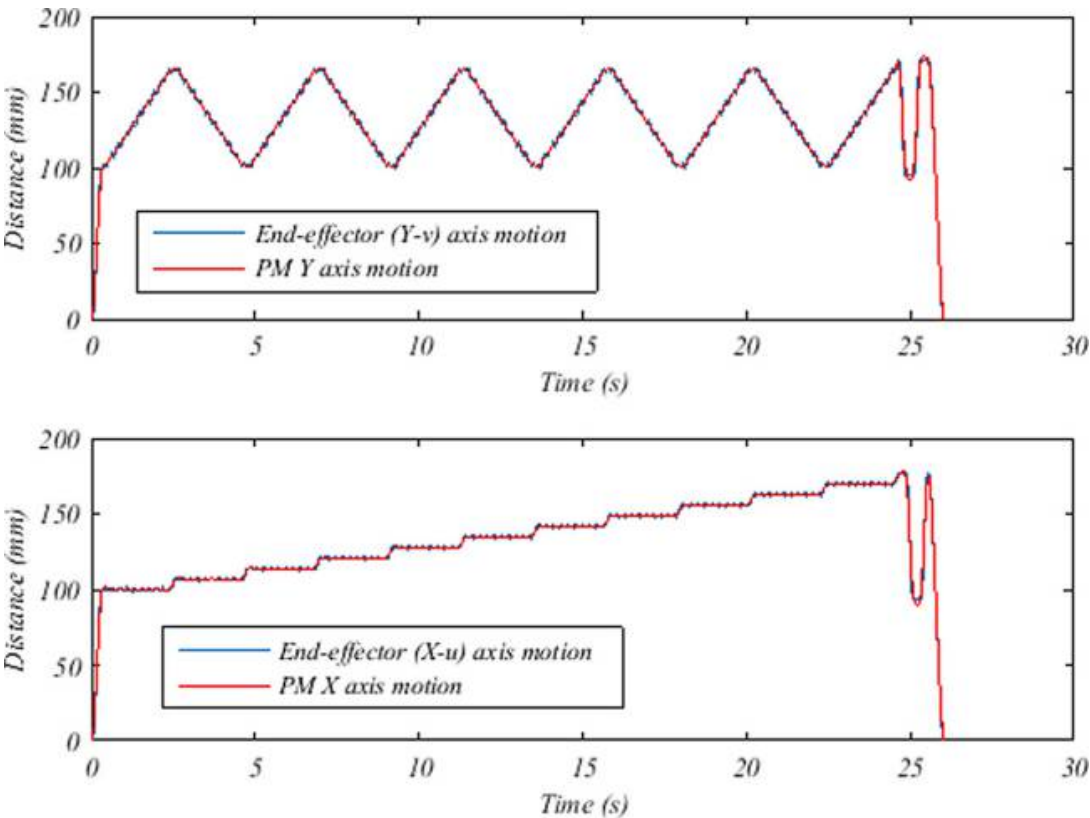

(a)


(b)

$9.81 \mathrm{~m} / \mathrm{s}^{2}$ and limited jerk. It takes about $119 \mathrm{~s}$ to complete the same task by using the motion profile generated for the conventional machine, which is shown in Figure 9.

When the laser-cutting machine is operated as a conventional machine, the errors calculated by comparing the tool motion to its trajectory are calculated to be bounded approximately in the range of $\pm 0.5 \mathrm{~mm}$ for both axes. The error graphs are presented in Figure 10.

Another benchmark piece is cut with the proposed algorithm, which has relatively more complex contours that consist of a double-headed eagle shape with crescent and star. The end-effector trajectory generated for benchmark cut is illustrated with blue lines and trajectory for the macro mechanism is represented with red lines in Figure 11. It takes about $82.53 \mathrm{~s}$ to complete the task by using the motion profile generated for the conventional machine and $13.50 \mathrm{~s}$ with the proposed method.

The pictures of manufactured workpieces for the 100 circle part and eagle with the crescent-star part are shown in Figure 12. These pieces are cut in the real production environment by using the motion profiles that are provided in Figures 5 and 11. 
Figure 7 Calculated motion of the micro mechanism's $(u-v)$ axes
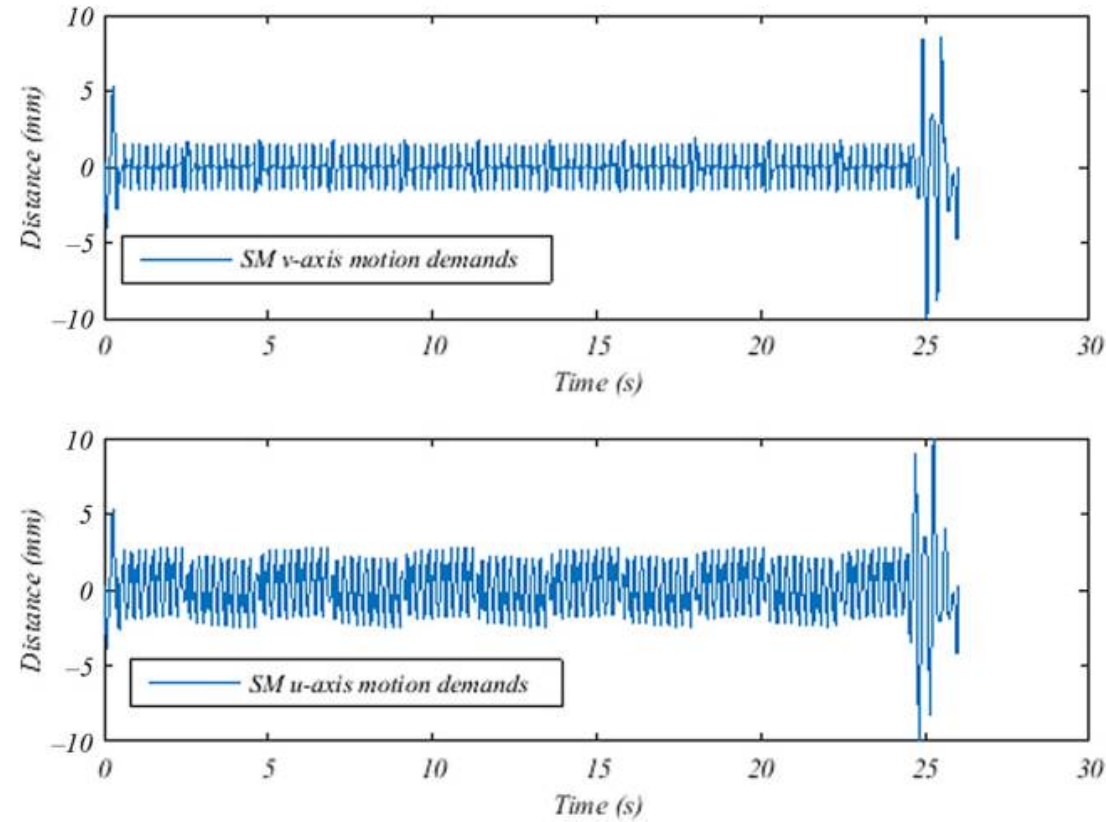

Figure 8 End-effector trajectory tracking error in $x$ - and $y$ - axes
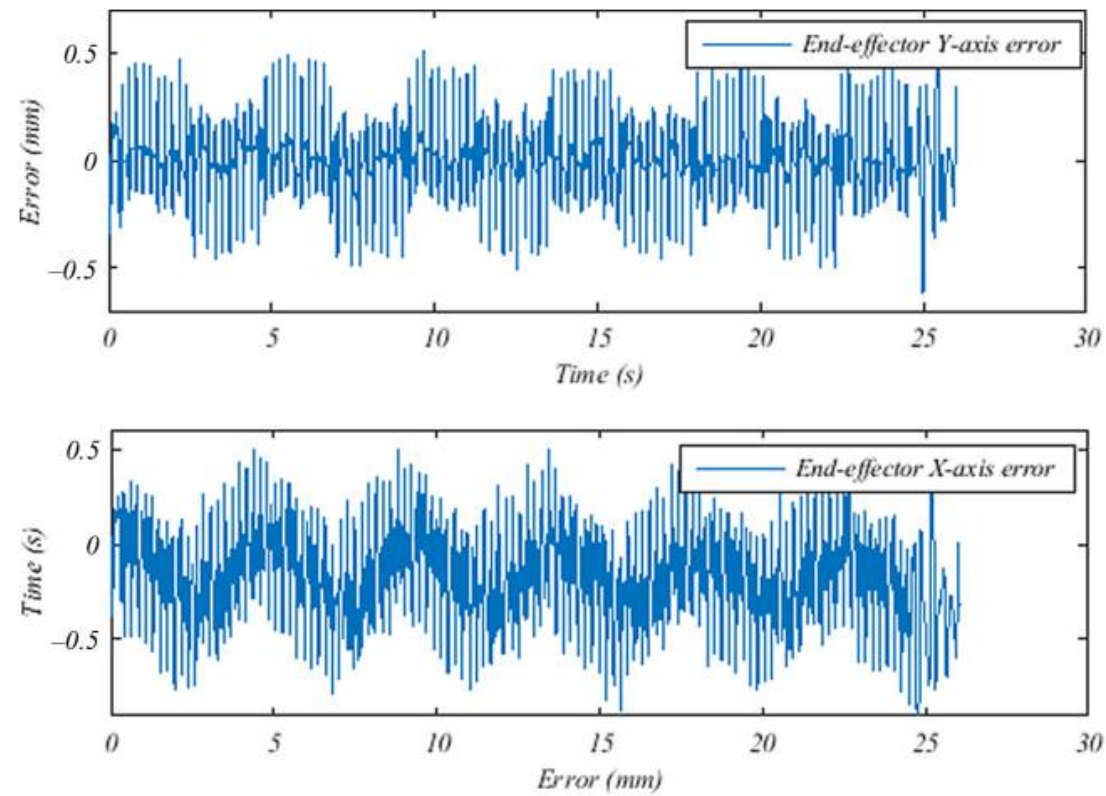

\section{Discussions and conclusions}

In this work, a new method for trajectory planning to reduce the task completion duration of a planar macro-micro mechanism laser-cutting machine is proposed. The novelty of this new method is that it does not have any practical limitations, as the trajectories are planned with respect to the physical limitations of the laser cutting machine, i.e. limited jerks. The new method deals with practical limitations by integrating an online trajectory generation with the offline method, and it is designed to be used as a post-processor in the industrial CNC systems.
The planar macro-micro mechanism laser-cutting machine, which is constructed within the same funded project, is used in experimentation. A benchmark workpiece is chosen to have relatively larger number of small circular cuts. The experiment to cut out the workpiece is carried out with both macro-micro mechanism machine and conventional machine. The results indicate that with the new trajectory planning algorithm the same piece can be cut out in less than a third of the duration it took to cut it out with the conventional machine. It can be argued that the precision of the operation can be improved especially by tuning the controller of the macro mechanism's 
Figure 9 Motion profile generated for conventional machine's axes
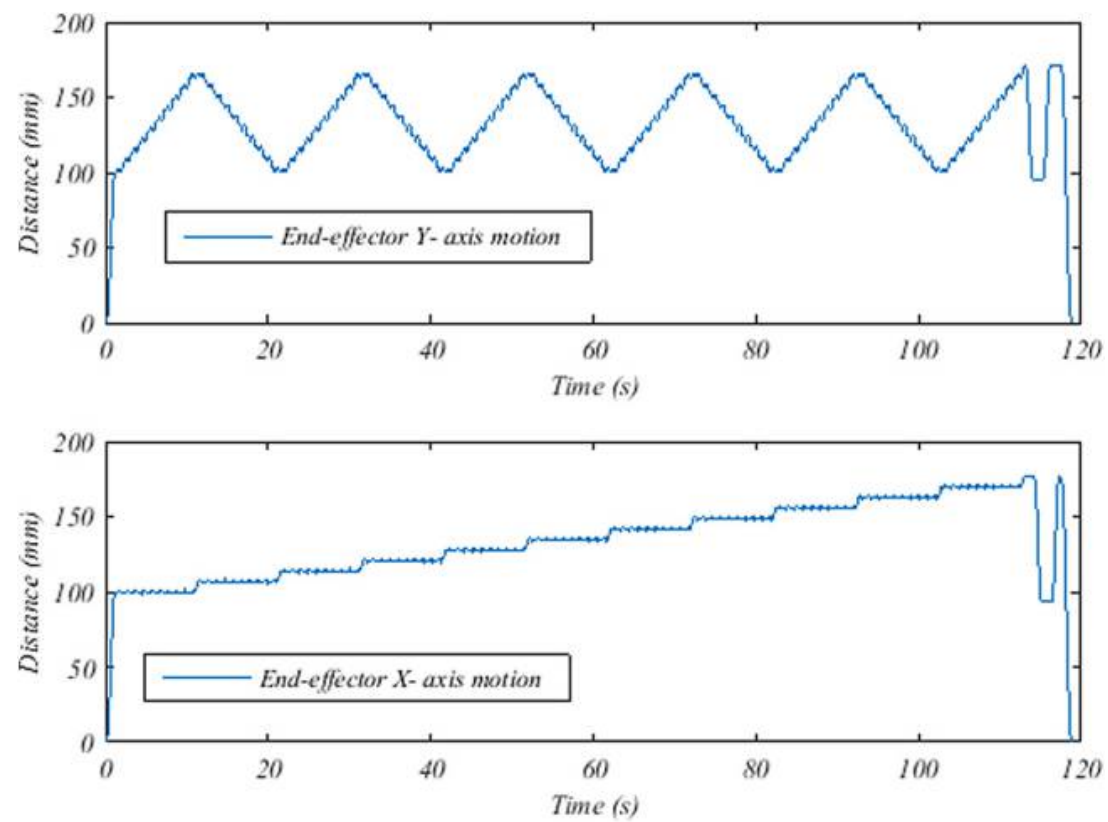

Figure 10 Tool trajectory tracking error along $x$ - and $y$-axes of the conventional machine
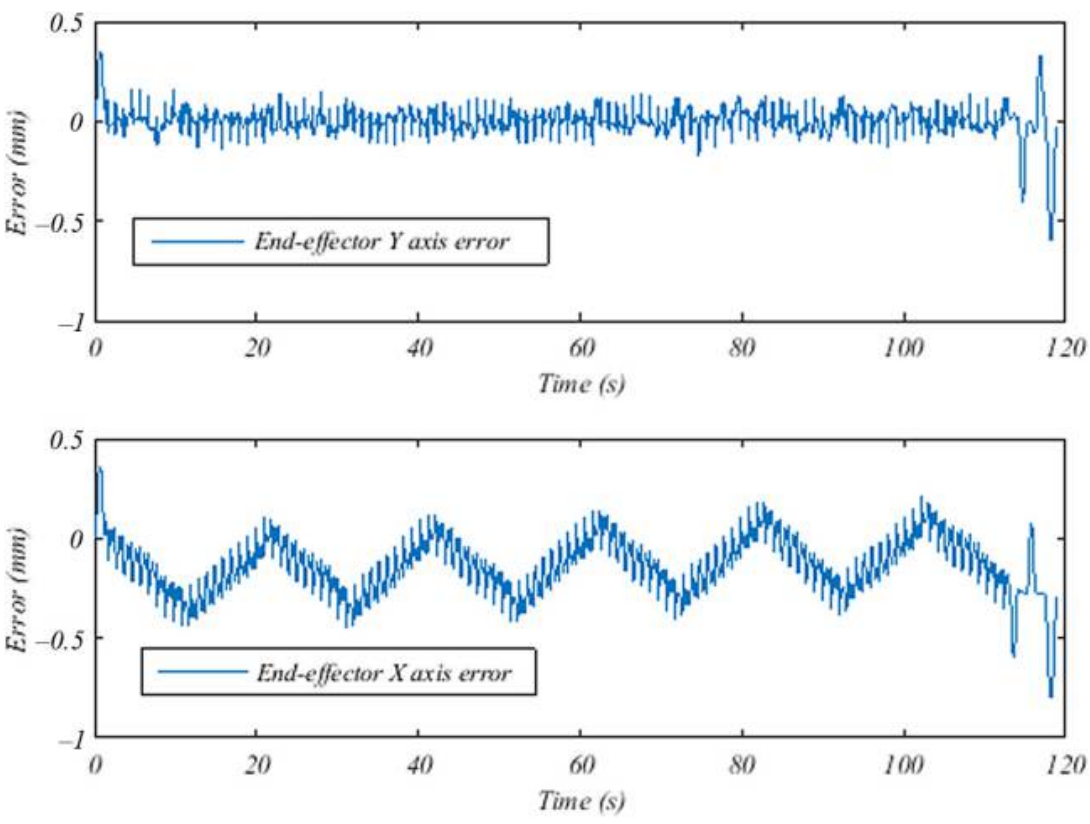

$x$-axis actuator. However, the important observation as a result of the tests is that the error range is about the same for both types of operation while the macro-micro mechanism machine decreased the task completion duration drastically.

The macro-micro mechanism machine developed in this study takes advantage of the larger workspace of the macro mechanism and the agility of the micro mechanism. The proposed concept including the machine and the algorithm can be used in CNC systems that have relatively large workspaces and manufacture workpieces, which have trajectories with relatively higher amount of smaller-radius curvature and/or small distance motions. In such CNC systems, time to reach maximum manufacturing speed gains importance to complete the manufacturing job in shorter duration. On the other hand, the maximum manufacturing speed is determined by the type of manufacturing (laser cutting, conventional machining processes, additive manufacturing) and the workpiece properties (material, thickness, etc.). The proposed concept does not improve or degrade the maximum manufacturing speed of the process but reduces the total task completion time.

During higher acceleration motions of the micro mechanism, deformation of the links is observed due to the relatively larger inertial properties of the machining tool (e.g. a laser head). For 
Figure 11 Motion trajectories generated for benchmark cut with the eagle and the crescent-star shaped piece

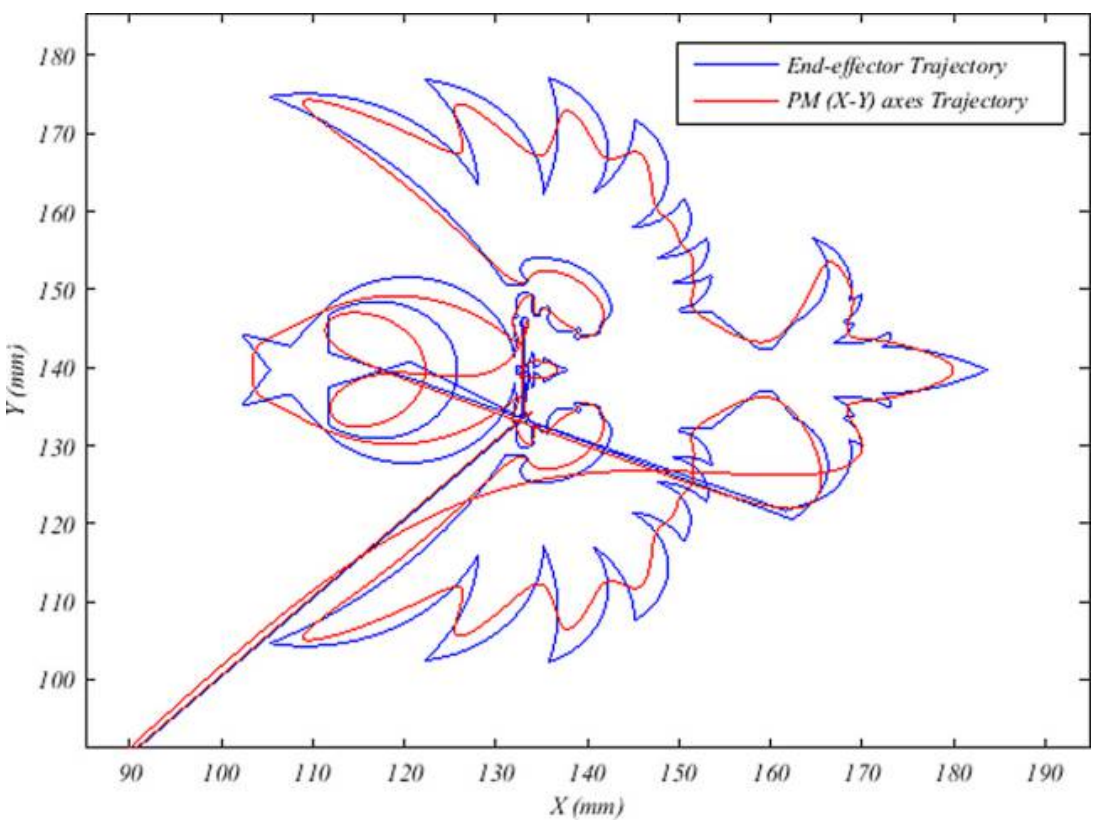

Figure 12 Pictures of manufactured benchmark parts in real production environment by using the proposed method

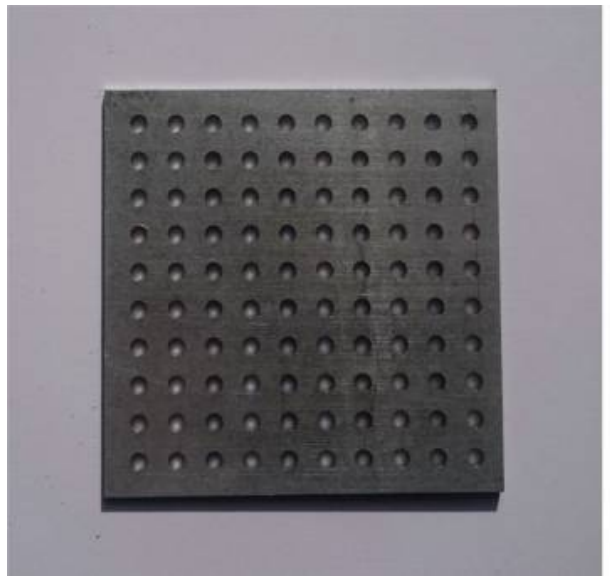

future work; stiffness model of the micro mechanism can be included in the trajectory planning algorithm to increase the accuracy and repeatability of the machining process.

\section{References}

Battheu, C. (2011), "Manipulator of low inertia for laser cutting machines for flat sheet metals", Patent no: US20110017714.

Cardinale, M., Carpanzano, E., Carracoi, M., Chirico, M., Lacasella, A., Masotti, S. and Mininno, E. (2007), "Method for managing systems, provided with redundant actuators", Patent no: US20070040527.

Chen, J.L., Liu, J.S., Lee, W.C. and Liang, T.C. (2002), "On-line multi-criteria based collision-free posture generation of redundant manipulator in constrained workspace", Robotica, Vol. 20 No. 6, pp. 625-636.

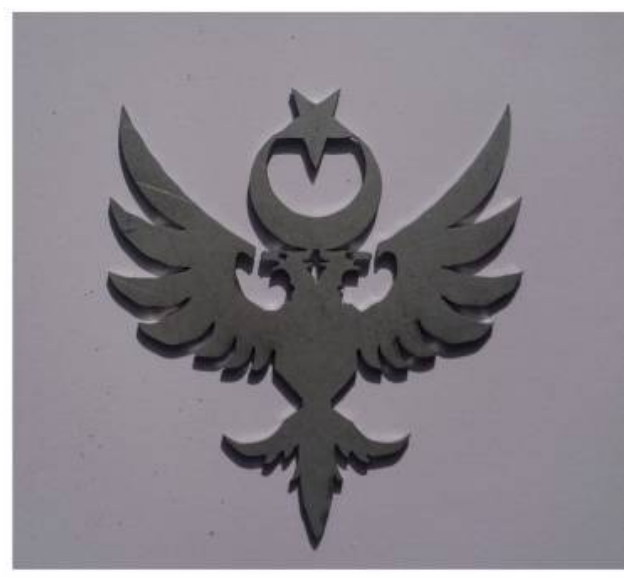

Dede, M.İ.C., Gezgin, E., Kiper, G., Mastar, E., Sı̆̆ırtmaç, T. and Uzunoğlu, E. (2014), "Design and analysis of a parallel mechanism for kinematically redundant hybrid planar laser cutting machine", The 16th International Conference on Machine Design and Production, Izmir, 30 June-3 July, pp. 810-822.

Hügel, H. (2000), "New solid-state lasers and their application potentials", Optics and Lasers in Engineering, Vol. 34 No. 4, pp. 213-229.

Khatib, O. (1991), "Reduced effective inertia in Macro-/ mini-manipulator systems", Fifth International Symposium on Robotics Research, Cambridge, pp. 279-284.

Kim, S.J., Koh, K., Boyd, S. and Gorinevskyi, D. (2009), " $1_{1}$ trend filtering", SIAM Review, Problems and Techniques Section, Vol. 51 No. 2, pp. 339-360.

Kiper, G., Dede, M.İ.C., Uzunoğlu, E. and Mastar, E. (2015), "Use of hidden robot concept for calibration of an over-constrained mechanism", The 14th IFToMM World 
Congress, Taipei, pp. 25-30, doi: 10.6567/IFToMM.14TH. WC.OS13.095.

Leibinger, P., Rauser, T. and Zeygerman, L. (2004), "Laser cutting machine with multiple drives", Patent no: US20040178181.

Maaroof, O., Gezgin, E. and Dede, M.İ.C. (2012), "General subtask controller for redundant robot manipulators", in 12th IEEE Int. Conf. on Control, Automation, Feju Island, pp. 1352-1357.

Marzwell, N.I., Uecker, D.R. and Wang, Y. (1994), "A force-controllable macro-micro manipulator and its application to medical robots", Technology 2003: The Fourth National Technology Transfer Conference and Exposition, NASA, Washington, Vol. 1, pp. 3-16.

Nagai, K. and Yoshikowa, T. (1994), "Impedance control of redundant macromicro manipulator", IEEE/RSF/GI International Conference on Intelligent Robots and Systems, Munich, pp. 1438-1445.

Nakamura, Y. (1991), Advanced Robotics: Redundancy and Optimization, Addison-Wesley Publishing Company, Melbourne.

Quan, B.T., Huang, J., Harada, M. and Yabuta, T. (2006), "Control of a macro-micro robot system using manipulability of the micro robot", FSME International Fournal Series C Mechanical Systems, Machine Elements and Manufacturing, Vol. 49 No. 3.

Sadigh, M.J. and Salehi, A. (2004), "A robust closed loop control for fast maneuvers of micro-macro manipulators", International Astronautic Conference Vancouver (Canada), Vancouver, Paper No. IAC-04-IAF-A., 5.04.

Sartorio, F. (2004), "Machine tool and manipulator devise adapted to be mounted on such machine", Patent No: US, 2004/0025761 A1.
Seraji, H. (1991), "Task options for redundancy resolution using configuration control", IEEE Conference on Decision and Control, Brighton, Vol. 3, pp. 2793-2798.

Sharon, A., Hogan, N. and Hardt, D.E. (1993), "The macro/ micro manipulator: an improved architecture for robot control", Robotics and Computer-Integrated Manufacturing, Vol. 10 No. 3, pp. 209-222.

Steen, W.M. and Mazumder, J. (2010), Laser MaKhterial Processing, 4th ed., Springer, London.

Tatlicioglu, E., Braganza, D., Burg, T.C. and Dawson, D.M. (2009), "Adaptive control of redundant robot manipulators with subtask objectives", Robotica, Vol. 27 No. 6, pp. 873-881.

Uzunoğlu, E., Dede, M.İ.C., Kiper, G., Mastar, E. and Sı̆̆ırtmaç, T. (2014), "Trajectory planning of redundant planar mechanisms for reducing task completion duration", Advances on Theory and Practice of Robots and Manipulators, Mechanisms and Machine Science, Springer, Dordrecht, Vol. 22, pp. 215-223, ISBN: 978-3-319-07057-5.

Uzunoğlu, E., Dede, M.İ.C. and Kiper, G. (2015), "Trajectory planning for a redundant planar laser-cutting machine with macro-micro manipulation", The 14th IFToMM World Congress, Taipei, 25-30 October, doi: 10.6567/IFToMM.14TH.WC.OS13.094.

Yoshikawa, T. (1984), "Analysis and control of robot manipulators with redundancy", Proceedings of First International Symposium on Robotics Research, MIT Press, Cambridge, pp. 735-748.

\section{Corresponding author}

Mehmet Ismet Can Dede can be contacted at: candede@ iyte.edu.tr

For instructions on how to order reprints of this article, please visit our website: www.emeraldgrouppublishing.com/licensing/reprints.htm Or contact us for further details: permissions@emeraldinsight.com 